Abstract 680 Table 1 Demographic and clinical features of the 57 included patients

\begin{tabular}{|c|c|c|c|}
\hline & Mean/median & & $\%$ \\
\hline Age (years)* & $60.2(11.9)$ & & \\
\hline BMI $\left(\mathrm{kg} / \mathrm{m}^{2}\right)^{* *}$ & $28.6(10.3)$ & & \\
\hline \multirow[t]{2}{*}{ Menopause } & & No & 28.1 \\
\hline & & Yes & 71.9 \\
\hline \multirow[t]{2}{*}{ Arterial hypertension } & & No & 57.9 \\
\hline & & Yes & 42.1 \\
\hline \multirow[t]{2}{*}{ Diabetes mellitus } & & No & 82.5 \\
\hline & & Yes & 17.5 \\
\hline \multirow[t]{2}{*}{ Previous delivery } & & No & 12.5 \\
\hline & & Yes & 87.5 \\
\hline \multirow[t]{2}{*}{ Previous Tamoxifen intake } & & No & 96.5 \\
\hline & & Yes & 3.5 \\
\hline \multirow[t]{2}{*}{ Previous cervical surgery } & & No & 93 \\
\hline & & Yes & 7 \\
\hline
\end{tabular}

Abstract 680 Table 2 Tracer detection and migration for Tc99 and ICG/Blue dye

\begin{tabular}{|c|c|c|}
\hline Tracer & $\mathrm{N}$ & $\%$ \\
\hline \multicolumn{3}{|l|}{ SPECT-TC Tc99 detection } \\
\hline No detection & 7 & 15.2 \\
\hline Pelvic unilateral & 10 & 21.7 \\
\hline Pelvic bilateral & 29 & 63 \\
\hline \multicolumn{3}{|l|}{ ICG/Blue dye migration } \\
\hline No migration & 5 & 9.2 \\
\hline Pelvic unilateral & 11 & 20.4 \\
\hline Pelvic bilateral & 38 & 70.4 \\
\hline
\end{tabular}

24.6\% (Tc99 5.3\%; ICG 17.5\%; blue dye 1.8\%). Cervix was the only injection site into submucosa and stroma.

In $89.5 \%$ of the patients, tracer migration was observed. Only 6 patients $(10.5 \%)$ had no migration. In table 2, detection and migration data of the tracers are shown.

One hundred forty-four SLNs were detected. Right side $(52.8 \%)$ was slightly more frequent than left side (47.2\%). Most SLNs were located in external iliac area $(40.5 \%)$, followed by iliac bifurcation (25.3\%), obturator fossa $(17.7 \%)$ and common iliac (13.9\%).

In three patients isolated tumor cells were detected (5.2\%) and one patient had macrometastases (1.8\%). 93\% of the patients had no pathological findings in SLNs.

Age was significantly higher in the no migration/no detection group in both Tc99 (70 vs 58.5 years; $\mathrm{p}=0.01$ ) and ICG/blue dye (72.5 vs 59.4 years; $\mathrm{p}=0.034)$. No migration/detection differences were detected for other patient's features.

Conclusion* A combined tracer technique is an effective method to detect SLNs in low-risk endometrial cancer to check lymphatic spread. Older women in our series have lower tracer migration/detection.

\section{INCIDENCE OF NODAL AND ISOLATED AORTIC METASTASES IN PATIENTS WITH SURGICALLY STAGED ENDOMETRIOID ENDOMETRIAL CANCER}

I Jaunarena*, R Ruiz, M Gorostidi, J Cespedes, D Del Valle, P Cobas, A Lekuona. Donostia Hospital, Obstetrics and Ginecology, San Sebastian, Spain

\subsection{6/ijgc-2021-ESG0.180}

Introduction/Background* Our objective was to describe the incidence of lymph node metastasis in patients with surgically staged endometrioid-type endometrial cancer in Donostia Hospital and evaluate the presence of isolated aortic metastasis. We believe, based on recent literature and our experience, that the number of lymph nodes involved in the aortic area is higher than traditionally reported.

Methodology A prospective observational study was conducted between 13 June 2014 and 31 December 2020 with 333 patients that underwent laparoscopic surgery for endometrial cancer at our institution. In all low, intermediate, high-intermediate and high ESGO/ESTRO/ESP 2020 prognostic risk cases, we performed sentinel lymph node (SLN) biopsy with dual cervical and fundal indocyanine green injection. All SLNs were processed with an ultrastaging technique. A total of 152 patients also underwent total pelvic and paraaortic lymphadenectomy.

Result(s)* The detection rates were as follows: 94\% overall for SLNs; 91.3\% overall for pelvic SLNs; 70.5\% for bilateral SLNs; $67.2 \%$ for paraaortic SLNs, $53.6 \%$ in 3 areas. A median of one aortic node and two pelvic nodes were removed. We detected positive SLNs in 56 patients $(16.8 \%$ of total cases); both pelvic and aortic SLNs were positive in 10 cases (representing 3\% of the sample; $17.8 \%$ of the total number of patients with positive nodes), while only pelvic SLNs were positive in $32(9.6 \% ; 57.1 \%)$ and only aortic SLNs were positive in $14(4.2 \% ; 25 \%)$. Sorting by myometrial infiltration, $<50 \%$ and $>=50 \%$, aortic isolated SLNs were positive in 4 and 10, representing $7.1 \%$ and $17.8 \%$ of the total with positive nodes (Fischer-Test Statistically significant), respectively.

Conclusion* In our series, the incidence of isolated aortic nodal metastasis is high compared with published reports. SLN biopsy allows a high rate of aortic detection, identifying a non-negligible percentage of isolated aortic metastases. Aortic metastases in endometrial cancer are possible and we should not give up actively looking for them. The highest rate of isolated aortic metastases occurred in patients with high-risk tumors, although there were also cases in patients with lowrisk tumors.

\section{ADVANCED ENDOMETRIAL CANCER SURGERY: WHAT REALLY IMPACTS ON SURVIVAL?}

D Tsolakidis*, E Markopoulou, S Pitis, D Zouzoulas, C Zymperdikas, M Lioupis, V Korvesi, M Topalidou, E Timotheadou, A Papanikolaou, G Grimbizis. General Hospital of Thessaloniki 'Papageorgiou', 1st Department of Obstetrics and Gynecology

\subsection{6/ijgc-2021-ESG0.181}

Introduction/Background* Endometrial cancer is the most common gynecological malignancy and its incidence is increasing steadily. More than $10 \%$ of cases will present with advanced stage disease. In these patients, the role and the time of surgery needs further clarification.

Methodology Retrospective analysis of all advanced endometrial cancer cases treated in our department from 2012 to 2018 was performed. Demographic and clinical variables were collected. Charlson comorbidity index was used to assess comorbidity. The use of neoadjuvant chemotherapy and the type of cytoreductive surgery (primary or interval debulking), surgical variables and residual disease after cytoreduction (no macroscopic disease, optimal cytoreduction $<1 \mathrm{~cm}$, residual $>1 \mathrm{~cm}$ or unresectable disease) were reported. Tumor characteristics were retracted from pathology reports. Descriptive 
statistics were used to present patients' and disease characteristics, as well as Kaplan- Maier curves for disease free survival and overall survival curves were estimated.

Result(s)* Records from 45 patients with stage IIIC and IV endometrial cancer between 2012 and 2018 were retracted. In our records, $35(78 \%)$ patients were treated with primary debulking and $10(22 \%)$ with neoadjuvant chemotherapy and interval debulking, because of primary inoperable tumor. Patients were staged as IIIC1 (13/45) IIIC2 (13/45) and IVa $(1 / 45)$ and IVb (18/45). Complete or optimal debulking (primary or interval) was achieved in $31 / 45$ patients (69\%), while residual or unresectable disease was recorded in 14/45 (31\%). Median overall survival was 38 months. Specifically, median overall survival in patients with complete cytoreduction (RD $0)$ was $>96$ months and 41 months in patients with optimal cytoreduction $(\mathrm{RD}<1)$. However, patients with residual disease $>1 \mathrm{~cm}$ had a median overall survival of 7 months. RD $<$ 1 showed a 4,67 increased risk for death (p: 0.012) and $R>1$ showed a 12,2-fold $(p<0,01)$ increased risk for death compared to complete cytoreduction. Age and ca- 125 at diagnosis and Charlson comorbidity index did not seem to have an impact on survival.

Conclusion* Complete cytoreduction is the most important factor which influences survival in advanced endometrial cancer patients.

\section{IS THE SARCOMATOUS COMPONENT THE PROGNOSTIC 'DRIVING FORCE' IN EARLY-STAGE UTERINE CARCINOSARCOMAS?}

${ }^{1} \mathrm{~A}$ Rosati ${ }^{*},{ }^{2} \mathrm{~V}$ Vargiu, ${ }^{1} \mathrm{C}$ Certelli, ${ }^{1} \mathrm{M}$ Arcieri, ${ }^{3} \mathrm{E}$ Vizza, ${ }^{4} \mathrm{~F}$ Legge, ${ }^{2} \mathrm{~F}$ Cosentino, ${ }^{1} \mathrm{G}$ Ferrandina, 'F Fanfani, ${ }^{1} \mathrm{G}$ Scambia, ${ }^{1} \mathrm{G}$ Corrado. ${ }^{1}$ Agostino Gemelli University Policlinic, Roma, Italy; ${ }^{2}$ Gemelli Molise, Italy; ${ }^{3}$ Regina elena, Rome, Italy; ${ }^{4}$ Ospedale Miulli acquaviva delle fonti, Acquaviva delle Fonti, Italy

\subsection{6/ijgc-2021-ESG0.182}

Introduction/Background* Uterine carcinosarcomas (UCSs) are aggressive biphasic malignancies, with a high grade carcinomatous/epithelial component and a high grade sarcomatous/mesenchymal counterpart. Several studies identified the carcinomatous part as the main factor affecting the aggressive behaviour of UCSs. However, other studies reported that the sarcomatous component, especially the presence of heterologous elements, was associated with a worse prognosis. The prognostic 'driving force' is not completely clear for these kind of tumours. For this reason, the aim of our study was to evaluate the impact of the sarcomatous component (Homologous vs Heterologous) on the overall survival (OS) and progression-free survival (PFS).

Methodology This is a multicenter observational retrospective study conducted in patients with stage I and II UCSs.

Result(s)* Ninety-five women with histological diagnosis of early stage UCSs were retrieved: 60 (63.2\%) had tumors with homologous sarcomatous components, and 35 (36.8\%) with heterologous. Tumors with a sarcomatous heterologous component were significantly larger than the homologous $(T \geq 50$ $\mathrm{mm}: 82.9 \%$ vs $51.7 \%$, p-value $=0.002$ ) and presented more often lymph-vascular space invasion $(62.9 \%$ vs $25.9 \%$ respectively in patients with heterologous and homologous component, $p$-value $=0.001$ ). At univariate analysis, a stromal invasion $\geq 50 \%$, the presence of clear cell, serous or undifferentiated carcinomatous component, the heterologous sarcomatous component and the FIGO stage IB and II were shown to be variables with a statistically significant negative impact on PFS. Similarly, a depth of invasion $\geq 50 \%$, the heterologous sarcomatous component and the FIGO stage IB and II were statistically negative prognostic factors also concerning OS. At multivariate analysis, only the heterologous sarcomatous component was confirmed to be a statistically significant negative prognostic factor both on PFS (HR 2.362, 95\% CI 1.2074.623, p-value $=0.012$ ) and on OS (HR 1.950, 95\% CI 1.032$3.684, \mathrm{p}=0.040)$.

Conclusion* In conclusion, in our large series of UCSs, both carcinomatous and sarcomatous components played a role in tumor progression and patients' survival. However, only the sarcomatous component retained a statistical significance at the multivariable model suggesting its preeminent prognostic role in early stage UCSs.

\section{PROGNOSTIC INDICATORS OF MALIGNANT INTRALUMINAL CELLS FOR PATIENTS WITH ENDOMETRIAL CANCER}

${ }^{1} \mathrm{C}$ Griffiths ${ }^{*},{ }^{1 ; 2} \mathrm{M}$ Gabriel, ${ }^{3} \mathrm{~K}$ Totolos, ${ }^{1} \mathrm{~J}$ Barroeta, ${ }^{1} \mathrm{D}$ Warshal. ${ }^{1} \mathrm{MD}$ Anderson Cancer Center at Cooper, Camden, USA; ${ }^{2}$ Cooper University Hospital, Obstetrics and Gynecology, Camden, USA; ${ }^{3}$ Cooper Medical School of Rowan University, Camden, USA

\subsection{6/ijgc-2021-ESGO.183}

Introduction/Background* Hysterectomy with bilateral salpingo-oophorectomy with nodal analysis is the preferred primary management for the vast majority of patients with endometrial cancer. ${ }^{1}$ Microscopic evaluation of the fallopian tubes occasionally identifies free floating intraluminal cancer cells (FFICC) without an in situ or invasive tubal component. ${ }^{2}$ FFICCs have been recently reported in the literature to be associated with lower survival in high risk endometrial cancer histologies. $^{3}$ The mechanism of action proposed involves exfoliation through the fallopian tubes and spillage into the peritoneal cavity leading to widespread metastatic potential. ${ }^{4}$ Although the significance of peritoneal cytology has historically been questioned and since removed from surgical staging, a positive correlation for FFICCs has been noted among patients with positive washings..$^{5}$ Our study aims to determine the incidence of FFICC's, risk factors as well as their prognostic significance.

Methodology A retrospective analysis was performed including all patients at our institution with a diagnosis of endometrial cancer who underwent surgical management between the years 2015 and 2018. Demographic and histopathologic variables were collected including stage, grade, lesion size, histologic subtype, and cytologic analyses. All recurrences, treatment plans, date of follow up and date of death were documented Result(s)* A total of 481 patients were included. Median age was 63 (28-92) with median follow up of 32 months. FFICCs were identified in $14 \%$ of the total sample (endometrioid, $n=$ 55 versus non-endometrioid histologies, $n=13$ ). Patients who had a robotic vs abdominal hysterectomy had an increased risk of FFICCs with an odds ratio of $2.9(\mathrm{p}=0.016)$. Presence of FFICCs was not clearly associated with grade or stage. Positive cytology was associated with an odds ratio of 6.6 